\title{
Numismatisches zur Geschichte von Limbach-Oberfrohna
}

\author{
Ewald Hausmann
}

Seit 25 Jahren ist der „Tag der Sachsen“ Anlass für die Herausgabe einer Medaille, mit der jeweils die Stadt gewürdigt wird, die zum größten Volksfest des Jahres im Freistaat einlädt. Die Sächsische Numismatische Gesellschaft führt damit die nun schon lange Reihe der Medaillen mit Motiven Sächsischer Städte fort. Für die Festmedaille auf Limbach-Oberfrohna wählte der Dresdner Medailleur Ralf Exner eine Gebäudegruppe als Motiv, das Rittergut mit der Stadtkirche, in je einer Version aus Vergangenheit und Gegenwart, getrennt durch eine waagerecht geschwungene Linie. In der unteren Ebene wird nach einer Abbildung aus der Mitte des 19. Jahrhunderts das Gutshaus mit Wirtschaftsgebäuden wiedergegeben. Darunter erinnert ein Erntewagen an die früher für die Region bestimmende Rolle der Landwirtschaft. Im oberen Teil erscheint das Gebäude in renovierter Pracht als das jetzige Rathaus. Die Umschrift zitiert den Wahlspruch „SUCHET DER STADT BESTES.“ Auf der Rückseite trennt das drapierte Stadtwappen die Namen der Ortsteile, die gegenwärtig die Stadt Limbach-Oberfrohna bilden. Darunter finden sich der Ausgabeanlass mit dem Signet des Kuratoriums „Tag der Sachsen“ und das Datum der Veranstaltung sowie das Namenskürzel des Herausgebers SNG. Die Medaille existiert in den Materialien Feinsilber, Kaiserzinn und vergoldeter Bronze, alle im Durchmesser von 40 Millimeter.

Neben die Festmedaille tritt der „Sachsentaggroschen“ als kleines Souvenir. Mit ihm wird die alte Tradition der Auswurfmünzen wiederbelebt, mit denen frühere Münzherren Festteilnehmer und Publikum beglückten. Die etwa groschengroßen Kupferstücke sind für die Verteilung vom Festwagen aus gedacht. Auf das Auswerfen wird aus rechtlichen Gründen verzichtet. Die Vorderseite nimmt das Schmuckwappen der Stadt ein. In einer rechteckigen Kartusche steht darunter der Name der Stadt. Die Umschrift „Sachsentaggroschen 2016“ nennt den Ausgabeanlass. Im Abschnitt findet sich mit SNG der Namenskürzel des Herausgebers. Die Rückseite ist dem Sponsor der Ausgabe gewidmet, dem Autohaus Lohs. Vor der Fassade des Firmengebäudes fährt ein VW Beetle in das Bild. Damit wird die Prägung zum Souvenir auch für Besucher des zeitgleich stattfinden-
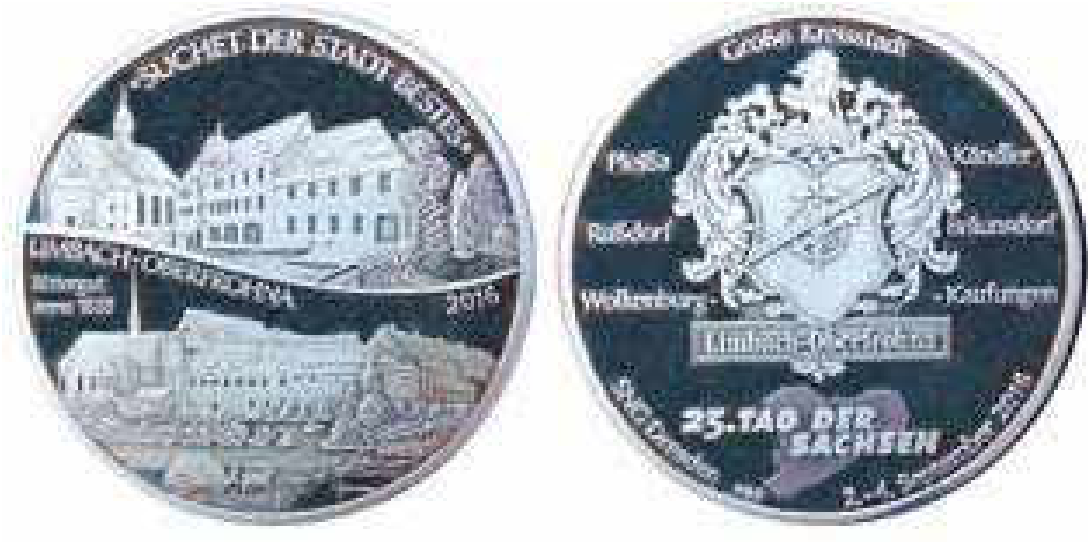

Festmedaille zum „Tag der Sachsen“, 2016 Fotos (S. 325-327): Ewald Hausmann
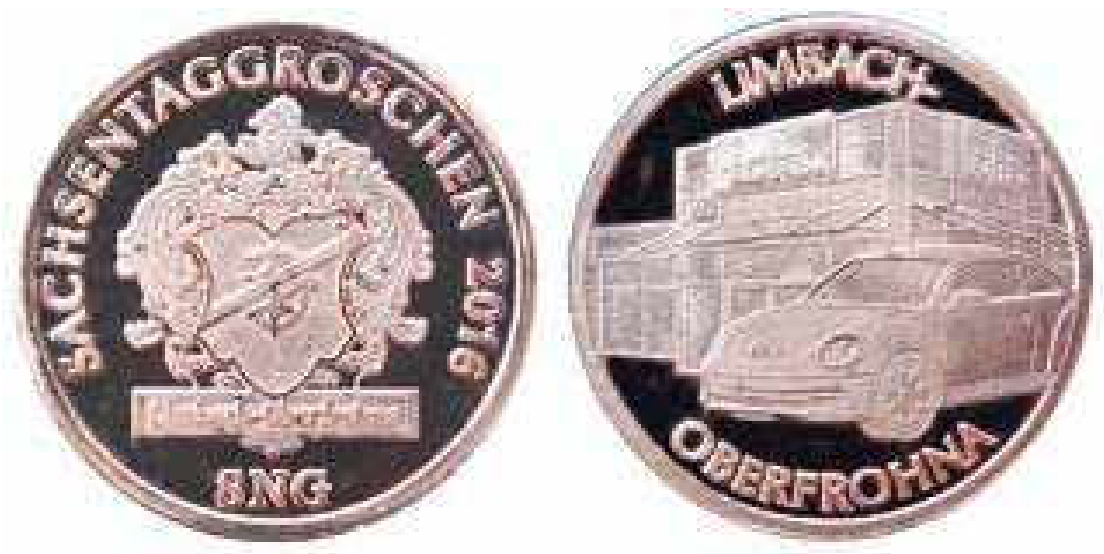

Sachsentaggroschen, 2016
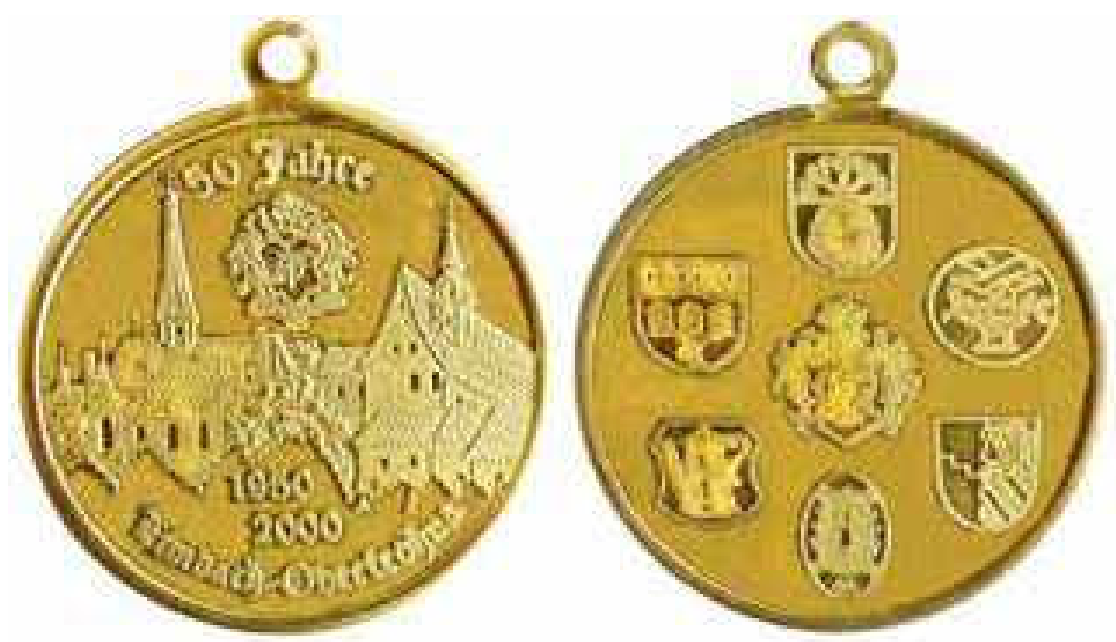

Medaille zum 50-jährigen Jubiläum der Stadt Limbach-Oberfrohna, 2000 

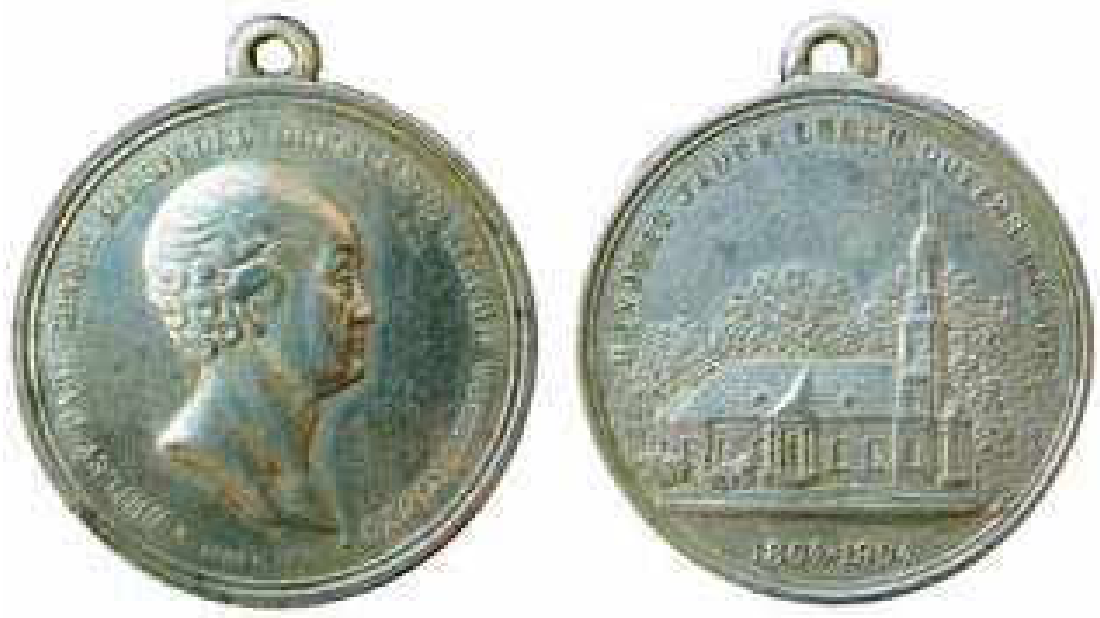

Medaille auf die Weihe der Mauritius-Kirche in Wolkenburg, 1904
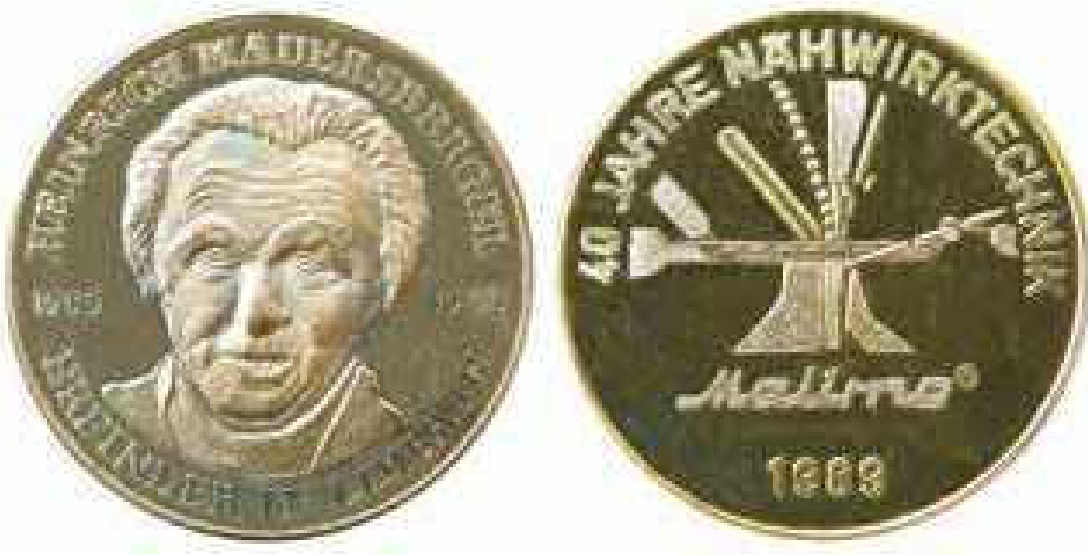

Medaille auf Heinrich Mauersberger, 1989

den Beetle-Treffens. Der Name der Stadt teilt sich die Flächen über und unter dem Bild. Ausführungen in Feinsilber und Kaiserzinn in geringer Auflage werden im Verkauf angeboten. Beide Medaillen kommen aus der 1. Dresdner Medaillen-Münze Glaser \& Sohn. Besucher können auf einem Klippwerk selbst Zinnronden zu Groschen schlagen.

Im Jahre 2000 war die erste der Stadt gewidmete Prägung erschienen. Bürgermeister Carsten Schmidt und sein Team veranlassten eine Würdigung der Vereinigung von Limbach und Oberfrohna vor 50 Jahren. Erstmals wurde die repräsentative Gebäudegruppe Rittergut mit Kirche als Motiv der Vorderseite gewählt. Darüber steht das Stadtwappen, darunter das Paar der Jahreszahlen 1950 / 2000. Die Umschrift nennt den Ausgabeanlass: oben „50 Jahre“ und unten „Limbach-Oberfrohna“. Auf der Rückseite ist das drapierte Gesamtwappen zu sehen, umgeben von den Wappen der sechs Dörfer, die die Ortsteile der Großen Kreisstadt bilden. Die angelötete Öse verweist auf die Bestimmung als Festmedaille. Die Herstellung übernahm die Firma Hans Simm in Renningen bei Stuttgart.

Der Gutschein ersetzt das 10-Pfennig-Stück der Vorkriegszeit aus einer Kupfer-Nickel-Legierung. die Mauritiuskirche in Wolkenburg einweihen konnte. Deren Bau hatte er veranlasst und finanziert. Die Vorderseite zeigt die Profilbüste des Grafen nach einer Plastik von Mattersberger, darunter die Lebensdaten „1737 - 1810“ und darüber die Umschrift „DETLEV CARL GRAF V. EINSIEDEL ERBAUER D. KIRCHE WOLKENBURG“. Auf der Rückseite wird die schönste klassizistische Dorfkirche Sachsens unter der Umschrift „HUNDERT JAHRE DURCH GOTTES GNADE“ vorgestellt. Im Abschnitt stehen die Jahreszahlen „1804 - 1904“. Die Medaille mit Trageöse besteht aus Zinn. Sie wurde offenbar als Festmedaille für ein breites Publikum konzipiert. Mit der Gestaltung waren vermutlich Künstler aus der Lauchhammerhütte betraut.

Im Jahre 1989 entstand eine Medaille zur Würdigung eines großen Sohnes der Stadt und seiner bahnbrechenden Erfindung. Anlass war das 40-jährige Jubiläum der Einführung des Malimo-Verfahrens. Die Frontalbüste des Geehrten steht auf der Vorderseite zwischen den Jahreszahlen 1909 und 1982, seinen Lebensdaten. Die Umschrift stellt ihn vor als „HEINRICH MAUERSBERGER / ERFINDER IN LIMBACH“. Frühere Mitarbeiter Mauersbergers fanden, der Text sei dringend notwendig, denn der Graveur habe das Gesicht arg verfremdet. Auf der Rückseite ist das Wirkprinzip des Verfahrens nach einer Zeichnung Mauersbergers dargestellt. Darunter steht das Bild des Warenzeichenverbandes Malimo. Die Umschrift nennt den Ausgabeanlass „40 JAHRE NÄHWIRKTECHNIK“. Den Abschnitt bildet das Datum des Ausgabejahres 1989. Die Ausgabe wurde von der Fachgruppe Numismatik Karl-Marx-Stadt veranlasst, aus der auch der Entwurf kam. Den Stempel schnitt Paul Schack in Waltershausen, in Tombak geprägt von der Fa. Bittner in Gotha.

Notgeldscheine aus den letzten Jahren des Ersten Weltkriegs und den ersten Jahren danach sind Zeugen der Ortsgeschichte von gänzlich anderer Art. 1917/18 führte zunehmender Mangel an Münzgeld zu ernsthaften Schwierigkeiten im Zahlungsverkehr. Gold- und Silbermünzen wurden für die Finanzierung der wenigen noch möglichen Importe gebraucht. Kupfer und Nickel, das Material der Kleinmünzen von

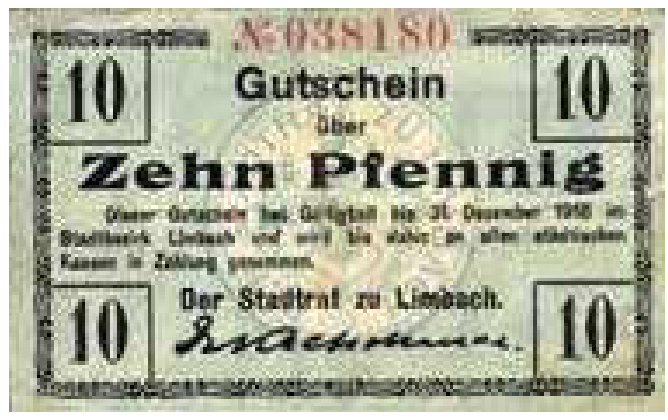


1 bis 20 Pfennigen, waren in der Rüstungsindustrie unentbehrlich und kamen deshalb für die Münzprägung nicht mehr in Frage. Das verunsicherte Publikum begann, die Münzen aus dem vertrauten Metall zu horten, wodurch die Verknappung des Kleingelds weiter zunahm. Prägungen in Stahl, Zink und Aluminium kamen erst spät in Gang. Wirksame Gegenmaßnahmen wurden auf der unteren Ebene organisiert: Kommunen und Händler gaben Gutscheine aus, deren Einlösung sie garantierten. In erster Linie ging es um Nominale unterhalb einer Mark.

Das nebenstehende Beispiel ist typisch: Herausgeber war der Stadtrat zu Limbach, der die Einlösung an städtischen Kassen bis zum Jahresende 1918 garantierte. Die Scheine akzeptierte man aber auch bei anderen Kassen, so dass sie teilweise erste nach mehreren Umläufen in der Stadtkasse eingeliefert wurden. Hergestellt wurden sie am Ort. Die Rückseite dieses Typs blieb unbedruckt.

Das Deutsche Reich hatte die immensen Kosten des Krieges mit dem Druck letztlich ungedeckter Banknoten und den Kriegsanleihen der Bevölkerung finanziert. Bei Kriegsende stand einem Übermaß an Geldzeichen ein Mangel an Waren gegenüber, was zu steigenden Preisen führte. Die Sieger forderten Reparationen von Deutschland im Betrag von etwa 160 Milliarden Goldmark - in Sachwerten, einschließlich Gold und Devisen. Hinzu kamen Besatzungskosten u. a. m. All dies heizte eine Superinflation an, mit der bis zu Ende November 1923 der Wert der Mark bis auf ein Billionstel der Goldmark sank. Zahlungen erforderten in immer kürzeren Zeitabständen zunehmend große Nominale. Die Reichsdruckerei kam dem Bedarf schließlich nicht mehr nach. Abhilfe musste wieder vor Ort geschaffen werden. Neben den Kommunen gaben nun auch Banken und Betriebe Zahlungsmittel aus. Die Ausgabe von Geldscheinen blieb aber nach wie vor nur der Reichsbank, den Landesbanken und der Reichskreditkasse erlaubt. Als Aushilfsmittel boten sich Schecks an. Kontoführende Geldinstitute wie örtliche Girokassen und überregionale Banken statteten ihre Kunden mit Scheckformularen aus, in denen z. T. schon Beträge vorgedruckt waren. Diese liefen um wie bares Geld. Wie die Banknoten der Reichsbank unterlagen sie dem Wertverfall durch die Inflation. Vom Konto des Ausstellers wurde der Betrag zu dem Kurs abgebucht, der am Tag der Einlösung galt.

Die Gestaltung der Schecks war vorwiegend sachlich gehalten und folgte überlieferten Mustern. Die Rückseite enthielt meist nur die Aufforderung „Nicht girieren“. Schecks von Geldinstituten aus den jetzigen Ortsteilen von Limbach-Oberfrohna erinnern an Verhältnisse in den schweren Jahre der Hochinflation.
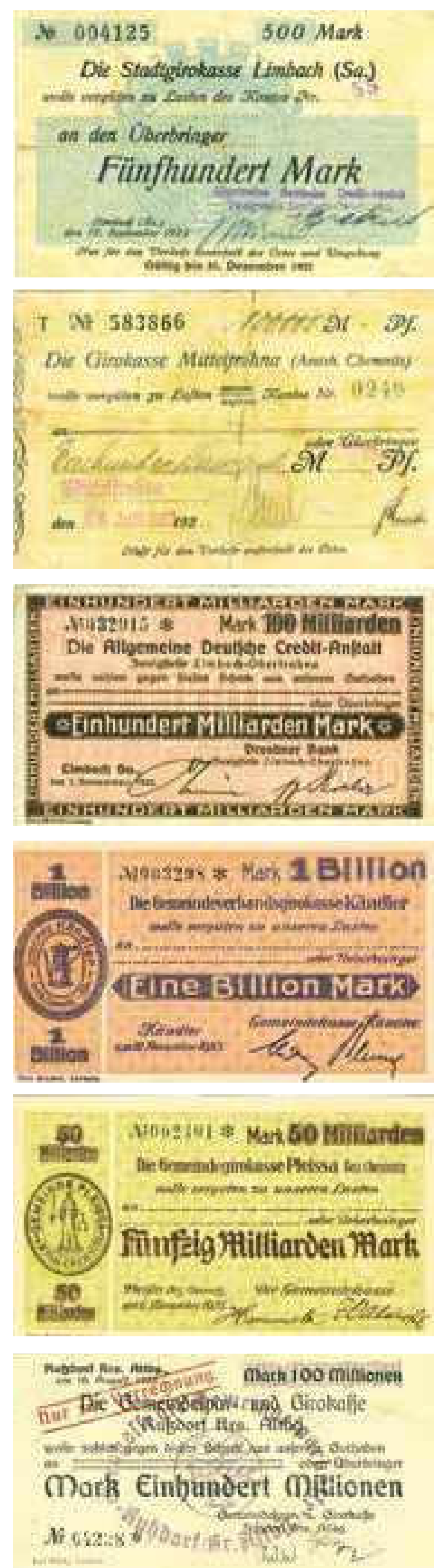

Die Stadtgirokasse Limbach stellte ihren Kunden Scheck-Vordrucke zur Verfügung, die wie Bargeld zirkulierten. Akzeptiert wurden sie auch in den Nachbarorten. Im Juli 1922 (Datum der Ausstellung) verfiel der Kurswert noch relativ langsam. Man konnte sich mit der Einlösung Zeit lassen.

Mittelfrohna hatte wie die anderen Dörfer eine eigene Girokasse. Deren Schecks hatte der Aussteller mit der Wertangabe zu versehen. Ihre Gültigkeit war auf den Ort beschränkt. 1923 fiel der Kurs der Mark so rasch, dass die Schecks möglichst umgehend eingelöst wurden. Eine Verrechnung zwischen einzelnen Girokassen konnte Tage dauern und Wertverlust mit sich bringen.

Die beiden Bankhäuser Dresdner Bank und Allgemeine Deutsche Credit-Anstalt setzten für gegenseitige Zahlungen Schecks mit vorgedruckten Wertstufen ein. Ihre Filialen waren für Limbach wie in Oberfrohna zuständig und nahmen den Namen der künftigen Stadt schon vorweg.

Die Girokasse Kändler wählte für den Scheck über die beeindruckende Summe eine etwas aufwändige Gestaltung. Am Ausstellungstag galt er 1 Billion Papiermark, 10 Tage später, auf dem Höhepunkt der Inflation, noch 150 Milliarden Papiermark bzw. ab 1. Dezember 0,15 Rentenmark.

Die Girokasse von Pleißa arbeitete ebenfalls mit vorgedruckten Wertzahlen.

In der Exklave Rußdorf stellte die Gemeindespar- und Girokasse vorgedruckte Schecks auf sich selbst aus.

Autor

Ewald Hausmann

Lichtenau 